\title{
Dissecting spectra with a SCALPEL
}

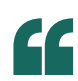

by combining

tailored NMR

experiments

and tensor

decomposition

methods we

may be able to

analyse much

more complex

mixtures

non-invasively
Analysis of complex mixtures is the cause of much trouble for even the most experienced chemists. The problem can be addressed by using separation techniques to simplify the sample being studied. However, this approach is in most cases tedious, time consuming and not always successful, as it may give a sample that is no longer representative of the original system. Now, Mathias Nilsson and co-workers have developed a new approach to dissecting the NMR spectra of unaltered complex mixtures. Writing in the Journal of the American Chemical Society, the team describe their method, called SCALPEL, which stands for spectral component acquisition by localized PARAFAC (parallel factor analysis) extraction of linear components. This is an apt description, because SCALPEL enables the different components of the original spectrum to be cut apart with high precision.

2D NMR spectroscopic techniques, such as total correlation spectroscopy (TOCSY) and diffusion-ordered spectroscopy (DOSY), can already resolve mixtures of limited numbers of species, in which the contributions to the overall spectrum are well separated or vary with time. Such constraints, however, tend to limit the use of these techniques and the amount of information that can be gained from their analysis, because of the overlapping spectral features of a many-component mixture. One possible approach to reduce the number of signals in a spectrum is to select a subset of spins from a small spectral region and label these with multiple types of behaviour (such as diffusion coefficient or relaxation times). If supported by suitable statistical analysis, then the spectra of individual components can be extracted without performing a physical separation.

"We design NMR experiments to give data tailored for statistical analysis based on powerful tensor decomposition methods; in this paper we used PARAFAC," explains Nilsson. "With experiments that yield data with the right structure, PARAFAC allows us to extract individual component spectra

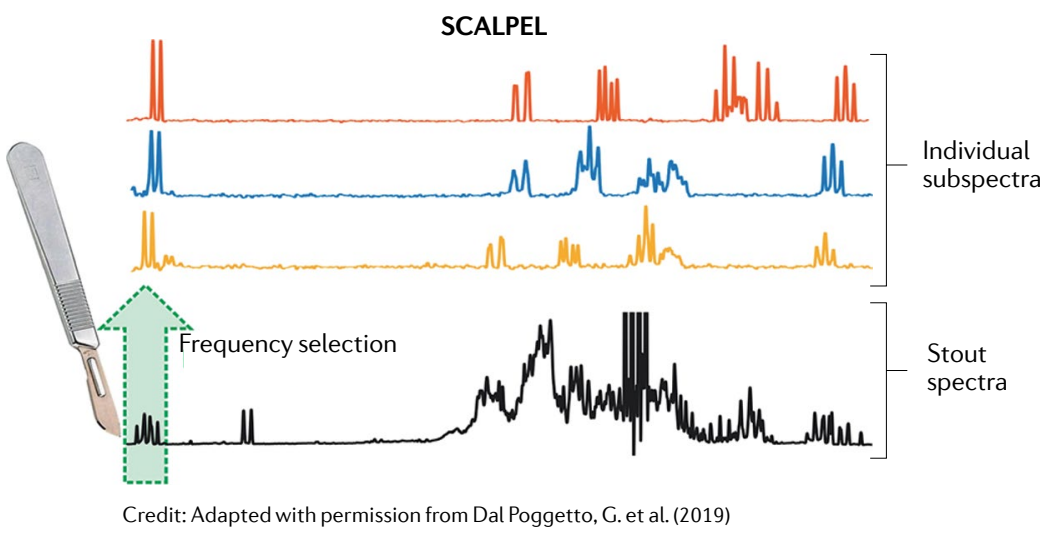

that are much easier to interpret. PARAFAC works best for mixtures with relatively small numbers of components, so we design our experiments to cut out one set of components at a time - hence the name."

Nilsson and co-workers demonstrate the concept by using SCALPEL to dissect the spectrum of a stout beer, in a region that features overlapping signals from ethanol and several carbohydrates. They developed a pulse sequence that labels spins based on their specific evolution during a delay, with either diffusion coefficient or relaxation time as a second spin label. By using diffusion encoding as the second data dimension, the spectral modes corresponding to the terminal $\beta$-glucose moieties of maltose/ maltotriose, lactose and free glucose were obtained, as these molecules have different sizes and therefore different diffusion coefficients. In their second experiment, the team used relaxation as the second spin label to successfully dissect the spectrum of an intact mixture of the disaccharides lactose and melibiose. In this case, SCALPEL enabled the identification of six distinct spin systems.

It is important to note that neither of the two labelling approaches could have given the same level of information if used in isolation. Nilsson concludes "This is an exciting proof of principle, showing that by combining tailored NMR experiments and tensor decomposition methods we may be able to analyse much more complex mixtures non-invasively. There are, indeed, potential applications in many areas of science, including metabolomics, natural product chemistry and drug discovery." 